\title{
CORRIGENDUM
}

\section{Association study of major risk single nucleotide polymorphisms in the common regulatory region of PARK2 and PACRG genes with leprosy in an Indian population}

Dheeraj Malhotra, Katayoon Darvishi, Manmohan Lohra, Himanshu Kumar, Chander Grover, Soni Sood, Belum SN Reddy and Ramesh NK Bamezai

European Journal of Human Genetics (2007) 15, 908. doi:10.1038/sj.ejhg.5201903

Correction to: European Journal of Human Genetics (2006) 14, $438-442$.

Since the publication of the above paper, the authors have identified a typographical error regarding the primer sequences for the forward and reverse primers of rs1040079 (SNP), where the reverse primer sequence is duplicated for the forward primer. The correct forward and reverse primer sequences are as follows:

Forward: 5-CTCGTGCCAGGAGGAAAAT-3

Reverse: 5-GGACTAAAGGGCATGGTGAG-3. 